\title{
INFLUENCIA DE LAS EMOCIONES EN LA COMPRA DE VINO POR ENOTURISTAS EN EL NUEVO MUNDO DEL VINO: EL CASO DE MÉXICO ${ }^{1}$
}

\author{
Lino Meraz Ruiz* \\ Universidad Autónoma de Baja California. México \\ https://orcid.org/0000-0002-7724-9176 \\ Virginia Margarita González Rosales* \\ Universidad Autónoma de Baja California. México \\ https://orcid.org/0000-0002-9070-0048 \\ Eduardo Raúl Díaz Gómez*** \\ CETYS Universidad. Tijuana. Baja California. México \\ https://orcid.org/0000-0003-0053-8751
}

\section{RESUMEN}

Un objetivo de las vinícolas es vender, pero se desconoce el rol de las emociones en la decisión de compra de vino. El objetivo de este estudio es analizar la importancia de emociones positivas, negativas y de vigilancia en la intención de compra de vino. Mediante una encuesta aplicada a 300 enoturistas en Valle de Guadalupe, se examina la influencia de tres estímulos: el vino, la vista a la vinícola, y una noticia sobre el vino. Los resultados sugieren que las emociones positivas tienen una mayor influencia en la intención de compra que las emociones o vigilantes. Conclusiones e implicaciones se discuten al final.

Palabras clave: Emociones; Enoturismo; Compra turística; Nuevo mundo del vino.

Influence of emotions on wine tourist purchase intention in a new world wine region: the case of Mexico

Fecha de recepción: 10 de abril de 2018

Fecha de aceptación: 14 de noviembre de 2018

*Facultad de Ciencias Administrativas y Sociales. Universidad Autónoma de Baja California. Boulevard Zertuche y Boulevard de los Lagos S/N Fracc. Valle Dorado C.P. 22890 Ensenada. BAJA CALIFORNIA (México). E-mail: lino.meraz@uabc.edu.mx,margarita.gonzalez@uabc.edu.mx.

**Escuela de Administración y Negocios. CETYS Universidad. Av. CETYS Universidad No. 4 Fracc. El Lago C.P. 22210 Tijuana, BAJA CALIFORNIA (México).E-mail: eduardo.diaz@cetys.mx.

1 Proyecto titulado: "Influencia de las emociones de enoturistas en la compra de vino en cuatro regiones del nuevo y viejo mundo del vino: México, España, Estados Unidos y Portugal”, no de referencia 414/7/N/8/3, financiado por la Universidad Autónoma de Baja California a través de la Coordinación de Posgrado e Investigación. 


\begin{abstract}
Wineries aim to sell, but the role of emotions on wine purchase intention is unclear. The objective of this study is to analyze the importance of positive, negative, and vigilant emotions on wine purchase intention. By analyzing survey results with 300 wine tourists, the influence of three stimuliare examined. These three stimuli are: one wine product, a visit to one winery, and a news report about the effects of wine. Results suggests that positive emotions have a greater influence on purchase intention than negative or vigilant emotions. Conclusions and implications are discussed.
\end{abstract}

Keywords: Emotions; Wine tourism; Tourist consumers; New world of wine.

\title{
1. INTRODUCCIÓN
}

El nuevo mundo del vino se refiere a las zonas colonizadas por europeos, donde se introdujo la producción del vino y en las que se ha generado un gran crecimiento de la industria vinícola en las últimas décadas, siendo América una de las regiones más representativas de este mundo del vino (Villanueva, 2014). La literatura ha puesto de manifiesto que la visión de la cultura del vino en ambos mundos, viejo y nuevo mundo del vino, difiere sustancialmente (Anderson, 2003) y el enoturismo puede exhibir diferentes características entre Europa y las nuevas regiones productoras (Charters y Ali-Knight, 2002).

Por otra parte, en ambos mundos del vino, el enoturismo se ha valorado como una manera estratégica de abordar directamente al cliente, para desarrollar marcas y vender in situ el vino (Howley y van Westering, 2008; Yeh yJeng, 2015). Así, el crecimiento del número de enoturistas en los últimos años ha despertado el interés de esta venta directa. A modo de ejemplo en California (Estados Unidos) en 2015 se estima que recibieron a 23.6 millones de turistas (WineInstitute, 2016), en Francia (Europa) 7.5 en 2010 (Atout France, 2010) y en Italia (Europa) 5 millones en 2008 (Romano yNatilli, 2010). Como se observa el enoturismo en estos dos mundos del vino es importante. Sin embargo, se desconoce si el comportamiento del turista es igual o diferente en estos destinos.

A este respecto, uno de los aspectos que se ha considerado tradicionalmente en la literatura sobre el comportamiento del turista es la diferencia entre culturas y género. Estas diferencias se han evidenciado en aspectos cognitivos, como son la percepción del precio (Stevens, 1992; Suh y McAvoy, 2005) o la evaluación de la calidad de los productos y servicios (Gilbert y Wong, 2003; Sultan y Simpson, 2000). No obstante, los estudios que han investigado la influencia de los aspectos afectivos en el comportamiento del turista comparando culturas son menos frecuentes (Chen y Huang 2017; Li et al., 2016) y los que examinan las diferencias en el comportamiento en las emociones del enoturista son prácticamente inexistentes.

Así, el objetivo de esta investigación, como primera fase de un proyecto comparativo entre regiones es identificar la influencia de las emociones que sienten los enoturistas 
en la apreciación de una marca de vino, la visita a la bodega y una noticia sobre el consumo de vino en un destino del nuevo mundo del vino (Baja California, México), analizando si el género es una variable moderadora de la influencia de las emociones en la intención de compra de vino en las bodegas. Resulta oportuno mencionar que en la literatura se ha demostrado que las emociones afectan directamente la compra de los productos (Bagozzi, 1997; Hirschman y Stern, 1999). También se ha determinado que existen tres tipos de emociones que afectan la compra (Pelegrín-Borondo et al., 2015; Penz y Hogg, 2011): las producidas por el producto que se evalúa (la oferta de vino), por el entorno en que se compra el producto (la visita a una bodega), y por aspectos ajenos tanto al producto como al entorno (la noticia sobre el vino).

En este sentido, el presente artículo ha sido estructurado en las secciones que se enumeran a continuación: Sección II, aborda la revisión literaria que propone un modelo teórico de las emociones de enoturistas. La Sección III plantea la metodología en que se enmarca el estudio. Sección IV, se discuten los resultados y se da respuesta a la comprobación de hipótesis. Finalmente, la Sección V presenta las principales conclusiones, limitaciones y futuras investigaciones.

\section{REVISIÓN DE LA LITERATURA}

En esta investigación se propone contrastar un modelo que analiza la influencia de las emociones, que sigue el enfoque de componentes (Russell, 2003; Scherer, 2005), producidas por el vino (producto), la visita a la bodega (entorno) y las noticias sobre el vino (ajenas al producto y al entorno) en la decisión de compra de vino en la bodega después de la visita, y cómo el género modera estas influencias (Figura 1). Bajo este enfoque se considera la existencia de ciertos factores para que exista una emoción (Pelegrín-Borondo et al., 2015), por ejemplo: estímulo emocional, atribución, evaluación cognitiva, reacciones fisiológicas, sensación de placer, procesos a corto plazo, entre otros.

\section{MODELO TEÓRICO DE LAS EMOCIONES DEL ENOTURISTA}

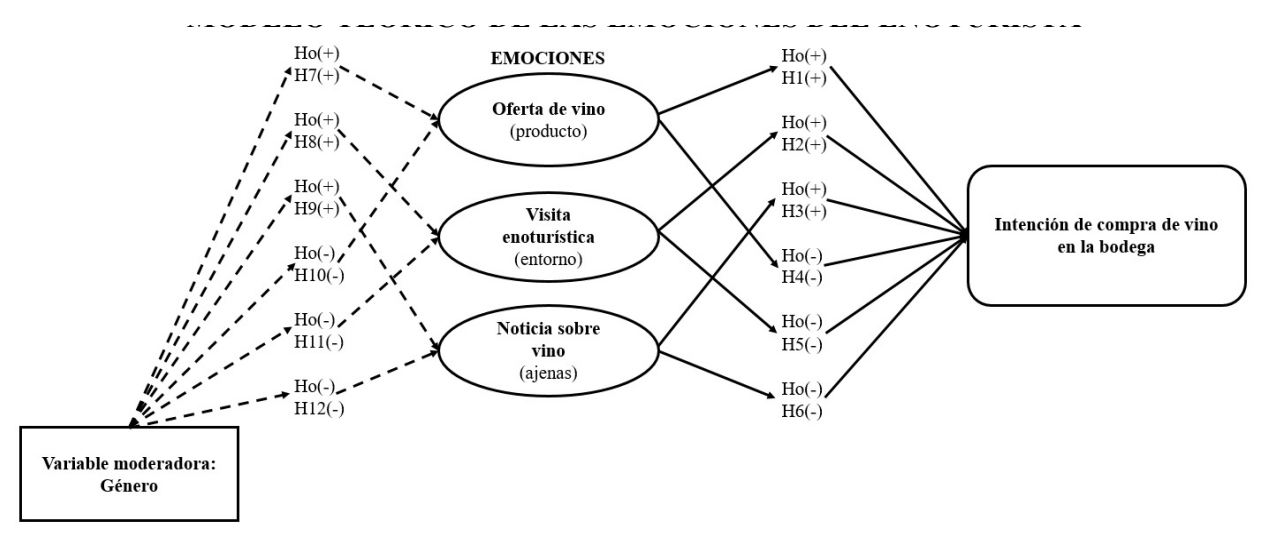


Dentro de este enfoque, la atribución se refiere al causante que genera el estímulo emocional que posteriormente va a producir la emoción (Weiner, 1985). De tal forma que para que exista una emoción ésta siempre debe ser causada por algo o alguien. En la compra de un producto se considera que puede haber tres tipos de causantes (PelegrínBorondo et al., 2016): el objeto que se está valorando (una oferta de vino), el entorno en el que se valora el objeto (la visita a una bodega) y causas ajenas a la propia compra (una noticia sobre el vino).

Independientemente del causante de la emoción, éstas pueden ser positivas o negativas (Blanco-González et al., 2013), aunque algunos autores han comentado la existencia de emociones que tienen alguna connotación positiva y negativa simultáneamente (Quintal et al., 2015). Los efectos de estas emociones positivas y negativas son diferentes (Sparks, 2007). En general, las positivas producen una tendencia a que el consumidor compre y las negativas provocan que se rechace el producto (Chang y Chieng, 2006; Lu et al., 2014).

\subsection{Influencia de las emociones generadas por la oferta de vino en la intención de compra de vino}

Respecto a las emociones producidas por el objeto que se está valorando, las investigaciones precedentes han demostrado que los productos que generan en el consumidor emociones positivas tienen mayor probabilidad de ser elegidos (Lee y Yun, 2015; Yeh et al., 2012), mientras que los que producen emociones negativas tienen mayor probabilidad de ser rechazados (Han et al., 2007; Sparks, 2007). Al referirse a las emociones producidas por los alimentos, Siegrist (2008) considera que los factores psicológicos son determinantes en la aceptación de los alimentos por los consumidores. Dentro de estos factores psicológicos varios investigadores han destacado la importancia de las emociones en la aceptación o rechazo de alimentos (Barrena y Sánchez, 2012; Teng, 2009).

En referencia a las emociones producidas por el vino, en varios estudios se ha demostrado que los consumidores sienten emociones positivas y negativas al catar vino (Horska et al., 2016) o al pensar en su consumo y han establecido el poder de estas emociones para segmentar a los consumidores (Silva et al. 2016). Por su parte, Dhar y Wertenbroch (2000) demostraron que los aspectos emocionales pueden conllevar a que una persona compre un vino sólo para probarlo, aunque en principio piense que sea una mala elección.

\subsection{Influencia de las emociones generadas por la visita a la bodega en la intención de compra de vino}

Tratándose de las emociones sobre el entorno de compra, la literatura ha demostrado que el ambiente en el que se realiza la compra genera emociones situacionales (Loebnitz et al, 2015) que afectan a la adquisición de los productos (Hamari, 2015). Es habitual que las empresas instauren en sus establecimientos de venta estímulos que generan emociones positivas que inducen a la persona a realizar compras o compras repetidas, como es el caso de una música agradable, colores u olores (Matute et al., 
2015). También se ha observado que aspectos negativos producidos en la tienda, como es la inexistencia de determinados artículos, produce emociones negativas que afectan a través de la satisfacción e imagen del establecimiento en el comportamiento de compra futura del cliente (Laroche et al., 1996). Algunos autores (Miao et al., 2011) aseguran que el ambiente en un restaurante de mala calidad generada por los clientes afecta a las emociones que sienten otros clientes y éstas afectan negativamente a la valoración del servicio del restaurante ( $\mathrm{Lu}$ et al., 2014), lo que pone en juego su asociación simbólica y propia congruencia (Das, 2014).

Respecto al enoturismo, Bruwer et al. (2013) infieren que es importante generar un ambiente de apego emocional hacia la bodega, su marca y su vino, pero sobre todo conocer la variación de necesidades y preferencias (Getz y Brown, 2006; Pratt, 2014). Por su parte, Yuan et al. (2008) reconocen la relevancia de los servicios prestados y el atractivo en la bodega para generar sentimientos positivos a los visitantes que afecten en su intención de comprar vino y volver a visitar la bodega (Bautzer, 2010; Ilicic y Webster, 2011; Zamora et al., 2006). En torno a estos factores, también se ha observado que los sentimientos asociados a la satisfacción y a la gratitud generan emociones positivas que amortiguan los efectos de las emociones negativas que el turista siente al verse en la obligación de comprar vino o souvenirs al concluir la visita (Bennet y Harrel, 1975; Kolyesnikova yDodd, 2008).

\subsection{Influencia de las emociones generadas por la noticia sobre el vino en la intención de compra de vino}

Ahora bien, atendiendo las emociones atribuidas por aspectos ajenos a la propia adquisición, algunos trabajos han demostrado que las emociones inducidas a través de lectura de textos o presentación de imágenes a los consumidores, antes de sus decisiones de compra, afectan su intención de compra (Labroo y Patrick, 2009; Griskevicius et al., 2010). Asimismo, se ha comprobado que estas emociones inducidas influyen en el proceso de valoración de las alternativas de compra (Buil et al., 2012; Kaefer et al., 2012) o afectando al procesamiento de la información (Wang et al., 2012).

Sobre las emociones en los turistas, Quintal et al. (2015) observaron que determinadas personas eligen hacer turismo como una forma de escape al estrés y problemas en el trabajo (Vanhove, 2005). Labroo y Rucker (2010) indican la existencia de emociones como: tristeza, enojo, vergüenza o ansia en los participantes de su investigación enmarcada por sentimientos de tristeza y felicidad. Por otro lado, en la literatura se ha puesto en tela de juicio que las noticias tienen la capacidad de generar emociones positivas y negativas en el comportamiento de las personas (Nie et al. 2015; Yu et al., 2015). Teniendo estos precedentes se proponen las siguientes hipótesis:

Ho: No existe relación entre intención de compra y emociones positivas sobre la oferta de vino.

H1: Existe relación entre intención de compra y emociones positivas sobre la oferta de vino.

Ho: No existe relación entre intención de compra y emociones positivas sobre la visita a la bodega. 
H2: Existe relación entre intención de compra y emociones positivas sobre la visita a la bodega.

Ho: No existe relación entre la intención de compra y emociones positivas sobre la noticia del vino.

H3: Existe relación entre intención de compra y emociones positivas sobre la noticia del vino.

Ho: No existe relación entre intención de compra y emociones negativas sobre la oferta de vino.

H4: Existe relación entre intención de compra y emociones negativas sobre la oferta de vino.

Ho: No existe relación entre intención de compra y emociones negativas sobre la visita a la bodega.

H5: Existe relación entre intención de compra y emociones negativas sobre la visita a la bodega.

Ho: No existe relación entre intención de compra y emociones negativas sobre la noticia del vino.

H6: Existe relación entre intención de compra y emociones negativas sobre la noticia del vino.

\subsection{El efecto moderador del género en la intención de compra de vino}

En lo que respecta a la moderación del género en la influencia de las emociones, Kolyesnikova et al. (2009) realizaron un estudio donde examinaron el conocimiento y motivaciones predictores del consumo de vino entre hombres y mujeres, establecen la existencia de diferencias entre género en la dimensión emocional asociada con la intención de compra, siendo las mujeres más comprometidas durante la compra a diferencia de los hombres que requieren un sentimiento de razón más fuerte. Por su parte, Lin (2015) encontró diferencias en las preferencias entre hombres y mujeres respecto a la selección de aperitivos entre universitarios, denotando una gran diferencia en los resultados, siendo las mujeres mucho más conscientes e influidas por los sentimientos en sus elecciones, a diferencia de la simplicidad de los hombres. Bastos et al. (2017), en un estudio sobre el consumo bebidas alcohólicas en jóvenes, conceptualizaron patrones similares de consumo en igualdad de género, lo que propicia la socialización y motivación para beber, estímulos positivos provocados por la predisposición y experiencia.

Referenciando el tema de amor a las marcas, los investigadores como Langner et al. (2014) observan diferencias significativas en las reacciones emocionales sobre ciertos productos, las mujeres tienden a ser neutrales, pero al paso del tiempo generan un vínculo con la marca, en cambio a los hombres les lleva años establecer un lazo afectivo. En el caso de Adidas, comparativamente, varios investigadores han encontrado diferencias y similitudes en la reacción emocional de los turistas ante los estímulos respecto al género (Collado et al., 2013; Gálvez et al., 2015; Gill et al., 2007). Al mismo tiempo, Ruiz et al. (2014), al establecer comparaciones en sus resultados con otros estudios (Dutta et al., 2007), el género juega un papel moderador en la intención de compra. Entonces, sobre la base de estos sustentos, se establecen las siguientes hipótesis: 
Ho: No existe relación respecto a emociones positivas sobre la oferta de vino entre participantes de género femenino y masculino.

H7: Existe relación respecto a emociones positivas sobre la oferta de vino entre participantes de género femenino y masculino.

Ho: No existe relación respecto a emociones positivas sobre la visita a la bodega entre participantes de género femenino y masculino.

H8: Existe relación respecto a emociones positivas sobre la visita a la bodega entre participantes de género femenino y masculino.

Ho: No existe relación respecto a emociones positivas sobre la noticia del vino entre participantes de género femenino y masculino.

H9: Existe relación respecto a emociones positivas sobre la noticia del vino entre participantes de género femenino y masculino.

Ho: No existe relación respecto a emociones negativas sobre la oferta de vino entre participantes de género femenino y masculino.

H10: Existe relación respecto a emociones negativas sobre la oferta de vino entre participantes de género femenino y masculino.

Ho: No existe relación respecto a emociones negativas sobre la visita a la bodega entre participantes de género femenino y masculino.

H11: Existe relación respecto a emociones negativas sobre la visita a la bodega entre participantes de género femenino y masculino.

Ho: No existe relación respecto a emociones negativas sobre la noticia del vino entre participantes de género femenino y masculino.

H12: Existe relación respecto a emociones negativas sobre la noticia del vino entre participantes de género femenino y masculino.

\section{METODOLOGÍA}

Para la comprobación de las hipótesis previamente planteadas y dar respuesta a los objetivos sobre el comportamiento del enoturista, se buscará realizar una comparación entre vinícolas del Nuevo y Viejo mundo del vino, en las regiones de California y Baja California para el primer bloque y en España y Portugal para el segundo. Como un primer esfuerzo en el estudio interregional es que se analiza a la primera bodega; la de Santo Tomás, la cual fue elegida por contar con las mismas características que sus homólogas en las demás zonas de estudio. Entre las prerrogativas para ser seleccionada se encuentra el hecho de haber sido fundada hace más de 100 años, el permitir y contar con una visitación planificada y estructurada para los turistas y el producir el mismo producto (Tempranillo) que en las bodegas de California y Europa.

Bodegas de Santo Tomás, fue fundada alrededor del año 1890, en Baja California, México. Exporta sus vinos a países en Europa, Norteamérica y Asia. Parte de sus esfuerzos se enfocan en el desarrollo del enoturismo y difusión de la cultura del vino mexicano. Bodegas de Santo Tomás es una de las bodegas más antiguas y visitadas en Baja California (Cervantes, 2012). El Estado de Baja California destaca por ser la zona que elabora la mayor producción de vino (95\% aproximadamente) del país, dada su amplia extensión territorial y condiciones climatológicas adecuadas (Trejo-Pech et al., 
2010). Esta zona tiene un amplio potencial turístico al estar situada a pocos kilómetros de la frontera con el Estado de California, Estados Unidos (Celaya, 2014). Los visitantes que recibe son similares a los turistas del Valle de Guadalupe en general. Es decir, los turistas de la zona tienden a visitar varias bodegas durante su estancia, por lo que el perfil de las personas que llevan a cabo recorridos en Santo Tomás es muy similar al perfil de visitantes en otras bodegas de la región.

En la bodega Santo Tomás se aplicaron 300 encuestas. Para efectuar el procedimiento de recogida de información se siguieron los siguientes pasos: (I) Antes de comenzar a rellenar el cuestionario se mostraba la oferta de vino, en la cual se incluyó información sobre la variedad de uva utilizada (100\% Tempranillo), descripción general de las características del vino (vino tinto de color rojo violáceo diáfano, frutos negros con notas de leña verde); el grado alcohólico (13,8\% Vol.); temperatura recomendada de consumo ( $16^{\circ}$ a $18^{\circ}$ Centígrados); crianza en barrica (8 meses en barricas de roble francés); precio de la botella (\$268 pesos mexicanos) (Figura 2). (II) Posteriormente se leía una noticia positiva sobre el consumo de vino ${ }^{2}$. (III) Finalmente, se le presentaba el cuestionario.

El instrumento de medición empleado en esta investigación es autoría de PelegrínBorondo (2017), el cual se compone de la escala PANAS que permite medir tres emociones generadas por el vino de la oferta, la visita a la bodega y una noticia general sobre el consumo de vino. Esta distrubucion genera nueve factores (tres emociones por cada uno de los tres estímulos). Dicha escala incluye ocho variables positivas, nueve variables negativas y tres variables de vigilancia: interesado, angustiado, excitado, disgustado, enérgico, culpable, asustado, hostil, entusiasmado, orgulloso, irritado, alerta, avergonzado, innovador, nervioso, decidido, vigilante, inquieto, activo y temeroso. Éste emplea un intervalo desde 0 puntos (no lo siente) hasta 10 puntos (lo siente intensamente). También se incluye una escala Likert de 11 puntos para medir la intención de compra del vino que incluye los ítems "Si pudiera, intentaría adquirir el vino de la oferta" y "Si pudiera, preveo que compraría el vino de la oferta".

Cabe mencionar que de la muestra obtenida el $100 \%$ fueron norteamericanos, $83.6 \%$ mexicanos y $16.3 \%$ estadounidenses, principalmente de California. El perfil de edad de la muestra obtenida es similar al de 340 turistas del estudio de Orta et al. (2016) en el mismo destino. En este artículo el rango de edad hasta los 39 años representaba el 50\%. En el presente estudio la muestra hasta los 39 años es del 66.3\%. La limitada información del estudio de Orta et al. (2016) no permite realizar más comparación. No obstante, en la presente investigación el porcentaje de mujeres es de $51.3 \%$ y $48.7 \%$ de hombres, el $81.7 \%$ de los turistas tienen estudios universitarios y $17.3 \%$ estudios secundarios.

2 Un estudio realizado con una muestra de 224 personas, a un grupo les dieron a beber vino tinto a diario en la cena, otro grupo vino blanco y otro agua mineral. Al final, las personas de vino tinto presentaron mejores niveles de colesterol y una disminución en diabetes. Los de vino blanco presentaron niveles saludables de triglicéridos que los bebedores de agua. 


\section{Figura 2 \\ OFERTA DE VINO MOSTRADA AL ENOTURISTA}

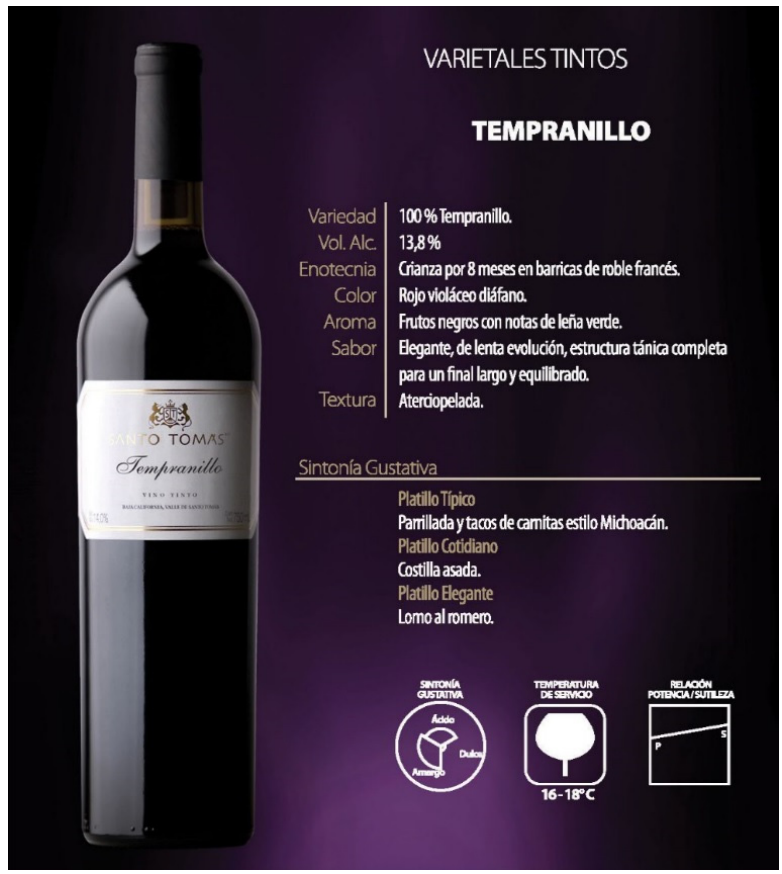

\subsection{Análisis estadístico}

Los nueve factores creados para realizar el presente estudio fueron desarrollados por el autor original con base a tres emociones por cada uno de los siguientes estímulos: la oferta del vino, la visita a la bodega y una noticia general sobre el vino. El análisis de componentes principales, llevado a cabo con apoyo del Statistical Package for the Social Sciences (SPSS) versión 21 , sugiere que los constructos, una vez ajustados, cumplen con los estándares de validez y fiabilidad para medir emociones positivas formuladas con base en las tres dimensiones mencionadas. El análisis factorial fue llevado a cabo con base al método de componentes principales utilizando el proceso de rotación varimax para reducir variables, fijando auto-estimaciones con valor de 1.0 o superior, e imponiendo una solución de nueve factores, en congruencia con el diseño original del instrumento. En el caso de emociones positivas de los participantes respecto a la oferta de vino (Cuadro 1), se empleó la medida KaiserMeyer-Olkin para medir la adecuación de la muestra que fue de.89, por encima del estándar de.60. Asimismo, el resultado de la prueba de esfericidad de Barlett fue estadísticamente significativo $(\mathrm{p}<.01)$ y el total de la variación explicada por el primer factor fue de $54.99 \%$.

En el segundo caso, emociones positivas en base a la visita a la bodega (Cuadro 2), repitiendo las pruebas anteriores, la medida de adecuación maestral Kaiser-Meyer-Olkin se estimó en.87 y la prueba de esfericidad de Barlett mostró valores estadísticamente 
significativos $(\mathrm{p}<.01)$. El total de la variación explicada fue de 50.63\%. En el tercer caso, emociones positivas respecto a la noticia sobre vino (Cuadro 3), los resultados fueron similares, con Kaiser-Meyer-Olkin de.90, Barlett con valores estadísticamente significativos $(\mathrm{p}<.01)$ y el total de la variación explicada por el factor correspondiente fue de $52.71 \%$. Como se muestra en el Cuadro 1, Cuadro 2 y Cuadro 3, los factores individuales muestran cargas factoriales aceptables y el alfa de Cronbach superó el estándar de.70.

\section{Cuadro 1}

EMOCIONES POSITIVAS SOBRE LA OFERTA DE VINO

\begin{tabular}{|c|c|c|c|c|c|}
\hline Emociones & $\mathbf{N}$ & $\begin{array}{c}\text { Factor } \\
1 \\
\end{array}$ & Comunalidades & $\mathbf{M}(\mathbf{D E})$ & $\begin{array}{c}\text { Alfa de } \\
\text { Cronbach }\end{array}$ \\
\hline Interesado & 300 & 0.6 & 0.36 & $8.02(2.63)$ & \multirow{8}{*}{0.881} \\
\hline Excitado & 300 & 0.66 & 0.43 & $4.38(3.82)$ & \\
\hline Enérgico & 300 & 0.71 & 0.51 & $4.94(3.71)$ & \\
\hline Entusiasmado & 300 & 0.79 & 0.63 & $6.56(3.38)$ & \\
\hline Orgulloso & 300 & 0.73 & 0.54 & $4.69(4.00)$ & \\
\hline Innovador & 300 & 0.78 & 0.61 & $5.46(3.96)$ & \\
\hline Decidido & 300 & 0.8 & 0.65 & $5.30(4.10)$ & \\
\hline Activo & 300 & 0.8 & 0.64 & $4.84(4.19)$ & \\
\hline
\end{tabular}

Nota: Las cargas del Factor 1 superan el estándar de.40 con comunalidades >.30. Fuente: Elaboración propia.

Cuadro 2

EMOCIONES POSITIVAS SOBRE LA VISITA A LA BODEGA

\begin{tabular}{|c|c|c|c|c|c|}
\hline Emociones & $\mathbf{N}$ & $\begin{array}{c}\text { Factor } \\
2 \\
\end{array}$ & Comunalidades & $\mathbf{M}(\mathbf{D E})$ & $\begin{array}{c}\text { Alfa de } \\
\text { Cronbach }\end{array}$ \\
\hline Interesado & 300 & 0.58 & 0.34 & $8.40(2.50)$ & \multirow{8}{*}{0.857} \\
\hline Excitado & 300 & 0.6 & 0.36 & $4.90(3.95)$ & \\
\hline Enérgico & 300 & 0.71 & 0.50 & $5.40(3.66)$ & \\
\hline Entusiasmado & 300 & 0.76 & 0.58 & $7.11(3.15)$ & \\
\hline Orgulloso & 300 & 0.64 & 0.42 & $5.19(4.01)$ & \\
\hline Innovador & 300 & 0.75 & 0.57 & $5.31(4.09)$ & \\
\hline Decidido & 300 & 0.78 & 0.62 & $5.03(4.13)$ & \\
\hline Activo & 300 & 0.79 & 0.63 & $5.06(4.22)$ & \\
\hline
\end{tabular}

Nota: Las cargas del Factor 2 superan el estándar de.40 con comunalidades >.30.

Fuente: Elaboración propia. 
Cuadro 3

EMOCIONES POSITIVAS SOBRE LA NOTICIA DEL VINO

\begin{tabular}{|c|c|c|c|c|c|}
\hline Emociones & $\mathbf{N}$ & $\begin{array}{c}\text { Factor } \\
\mathbf{3} \\
\end{array}$ & Comunalidades & M(DE) & $\begin{array}{c}\text { Alfa de } \\
\text { Cronbach }\end{array}$ \\
\hline Interesado* & 299 & 0.24 & 0.06 & $8.04(7.12)$ & \multirow{8}{*}{0.89} \\
\hline Excitado & 299 & 0.7 & 0.49 & $4.17(3.94)$ & \\
\hline Enérgico & 299 & 0.75 & 0.56 & $4.46(3.69)$ & \\
\hline Entusiasmado & 299 & 0.78 & 0.61 & $6.05(3.46)$ & \\
\hline Orgulloso & 299 & 0.76 & 0.58 & $3.75(3.81)$ & \\
\hline Innovador & 299 & 0.8 & 0.64 & $4.37(3.91)$ & \\
\hline Decidido & 299 & 0.78 & 0.61 & $4.22(4.08)$ & \\
\hline Activo & 299 & 0.8 & 0.64 & $3.83(4.09)$ & \\
\hline
\end{tabular}

Nota 1: Las cargas del Factor 3 superan el estándar de.40 con comunalidades $>.30$, con excepción de * que se elimina del análisis por contar con carga $<.40$.

Nota 2: Un error durante el proceso de captura de datos fue detectado y provocó que se eliminara la respuesta, razón por la cual la muestra se reduce de 300 a 299.

Fuente: Elaboración propia.

Respecto a las emociones negativas entre los participantes con base a su apreciación de la oferta de vino, la visita a la bodega y la noticia general del vino, se concluye que el instrumento soporta el rigor estadístico de manera semejante a las emociones positivas. El Cuadro 4, Cuadro 5 y Cuadro 6 sugieren que los resultados de la encuesta pueden considerarse válidos y con un aceptable nivel de consistencia interna. En el caso de emociones negativas respecto a la oferta de vino, la medida de adecuación muestral KaiserMeyer-Olkin arrojó un resultado de.87 al aplicarse a los datos de la encuesta. La prueba de esfericidad de Barlett tuvo importancia estadística $(\mathrm{p}<.01)$, y el total de la variación explicada por el cuarto factor fue de $57.14 \%$. El análisis arrojó una segunda iteración del factor 4, lo cual produjo que se eliminaran algunas variables (ver Cuadro 4).

\section{Cuadro 4}

EMOCIONES NEGATIVAS SOBRE LA OFERTA DE VINO

\begin{tabular}{|l|c|c|c|c|c|c|}
\hline Emociones & N & \multicolumn{2}{|c|}{ Factor 4 } & Comunalidades & M(DE) & $\begin{array}{c}\text { Alfa de } \\
\text { Cronbach }\end{array}$ \\
\cline { 1 - 6 } Angustiado & 300 & 0.7 & -0.35 & 0.62 & $.71(1.84)$ & \\
\cline { 1 - 5 } Disgustado & 300 & 0.78 & -0.38 & 0.76 & $.55(1.55)$ & \multirow{2}{*}{0.87} \\
\cline { 1 - 5 } Culpable & 300 & 0.73 & 0.09 & 0.55 & $.45(1.42)$ & \\
\cline { 1 - 5 } Asustado & 300 & 0.82 & -.14 & 0.69 & $.47(1.56)$ & \\
\hline
\end{tabular}




\begin{tabular}{|c|c|c|c|c|c|c|}
\hline Emociones & $\mathbf{N}$ & \multicolumn{2}{|c|}{ Factor 4} & Comunalidades & $\mathbf{M}(\mathbf{D E})$ & Alfa de \\
\hline Hostil* & 300 & 0.76 & 0.44 & 0.77 & $.42(1.38)$ & \\
\hline Irritado & 300 & 0.82 & 0.04 & 0.67 & $.36(1.15)$ & \\
\hline Avergonzado & 300 & 0.73 & -0.46 & 0.75 & $.26(1.01)$ & 0.87 \\
\hline Nervioso* & 300 & 0.69 & 0.3 & 0.57 & $.52(1.50)$ & \\
\hline Temeroso* & 300 & 0.73 & 0.49 & 0.77 & $.43(1.37)$ & \\
\hline
\end{tabular}

Nota: Las cargas del Factor 4 superan el estándar de.40 con comunalidades $>.30$, pero se eliminan las variables* por contar con una segunda carga $>.30$.

Fuente: Elaboración propia.

Las emociones negativas respecto a la visita a la bodega, que corresponde al análisis presentado en el Cuadro 5, el resultado de Kaise-Meyer-Olkin fue de.86, por encima del estándar de.60, la prueba Barlett arrojó valores significativos $(\mathrm{p}<.01)$, y el total de la variación explicada fue de $55.63 \%$. Los resultados sugieren validez y fiabilidad del instrumento de medición. En el siguiente caso de emociones negativas respecto a la noticia sobre el vino, Kaiser-Meyer-Olkin arrojó.85, por encima del estándar de.60, Barlett sugiere resultados significativos $(\mathrm{p}<.01)$, y el total de la variación explicada fue de $50.21 \%$. El nivel de correspondencia por factor y alfa de Cronbach se ilustra en el Cuadro 6.

\section{Cuadro 5}

EMOCIONES NEGATIVAS SOBRE LA VISITA A LA BODEGA

\begin{tabular}{|c|c|c|c|c|c|}
\hline Emociones & $\mathbf{N}$ & $\begin{array}{c}\text { Factor } \\
5\end{array}$ & Comunalidades & $\mathbf{M}(\mathbf{D E})$ & $\begin{array}{c}\text { Alfa de } \\
\text { Cronbach }\end{array}$ \\
\hline Angustiado & 300 & 0.56 & 0.31 & $.56(1.64)$ & \multirow{9}{*}{0.851} \\
\hline Disgustado & 300 & 0.64 & 0.41 & $.47(1.56)$ & \\
\hline Culpable & 300 & 0.75 & 0.56 & $.32(1.35)$ & \\
\hline Asustado & 300 & 0.82 & 0.67 & $.29(1.23)$ & \\
\hline Hostil & 300 & 0.75 & 0.56 & $.34(1.27)$ & \\
\hline Irritado & 300 & 0.72 & 0.52 & $.33(1.23)$ & \\
\hline Avergonzado & 300 & 0.7 & 0.49 & $.19(.82)$ & \\
\hline Nervioso & 300 & 0.61 & 0.37 & $.59(1.70)$ & \\
\hline Temeroso & 300 & 0.66 & 0.44 & $.36(1.19)$ & \\
\hline
\end{tabular}

Nota 1: Las cargas del Factor 5 superan el estándar de.40 con comunalidades >.30.

Fuente: Elaboración propia. 


\section{Cuadro 6}

\section{EMOCIONES NEGATIVAS SOBRE LA NOTICIA DEL VINO}

\begin{tabular}{|c|c|c|c|c|c|}
\hline Emociones & $\mathbf{N}$ & $\begin{array}{c}\text { Factor } \\
6\end{array}$ & Comunalidades & M(DE) & $\begin{array}{c}\text { Alfa de } \\
\text { Cronbach }\end{array}$ \\
\hline Angustiado & 300 & 0.56 & 0.31 & $.84(2.08)$ & \multirow{9}{*}{0.858} \\
\hline Disgustado & 300 & 0.61 & 0.37 & $.43(1.50)$ & \\
\hline Culpable & 300 & 0.76 & 0.58 & $.51(1.60)$ & \\
\hline Asustado & 300 & 0.84 & 0.7 & $.37(1.32)$ & \\
\hline Hostil & 300 & 0.68 & 0.46 & $.39(1.33)$ & \\
\hline Irritado & 300 & 0.75 & 0.56 & $.36(1.11)$ & \\
\hline Avergonzado & 300 & 0.74 & 0.54 & $.37(1.39)$ & \\
\hline Nervioso & 300 & 0.63 & 0.39 & $.81(1.99)$ & \\
\hline Temeroso & 300 & 0.75 & 0.56 & $.43(1.39)$ & \\
\hline
\end{tabular}

Nota: Las cargas del Factor 6 superan el estándar de.40 con comunalidades >.30.

Fuente: Elaboración propia.

Los resultados del análisis de componentes utilizado para medir las emociones de vigilancia de los participantes respecto a la oferta de vino, la visita a la bodega y la noticia sobre el vino muestran niveles bajos de validez y consistencia interna. En el primer caso sobre emociones de vigilante respecto a la oferta de vino, la prueba arroja un resultado de Kaiser-Meyer-Olkin de.59, por debajo del estándar de.60 y el alfa de Cronbach se encuentra por debajo del estándar de.70 (Cuadro 7). Resultados similares se identificaron en el segundo caso sobre emociones respecto a la visita a la bodega con un Kaiser-Meyer-Olkin de.54 y un alfa de Cronbach de.590 (Cuadro 8). Similarmente, para el tercer caso sobre emociones de vigilante respecto a la noticia sobre el vino, se calculó un Kaiser-MeyerOlkin de.60, apenas cumpliendo con el estándar, y un alfa de Cronbach de.629 (Cuadro 9). En este último, es posible presentar un argumento para aceptar la validez y fiabilidad del factor, pero se opta por tomar una postura conservadora y eliminar los tres últimos factores del instrumento y análisis posteriores.

\section{Cuadro 7}

\section{EMOCIONES VIGILANTE SOBRE LA OFERTA DE VINO}

\begin{tabular}{|c|c|c|c|c|c|}
\hline Emociones & $\mathbf{N}$ & Factor 7 & Comunalidades & $\mathbf{M}(\mathbf{D E})$ & Alfa de Cronbach \\
\hline Alerta & 300 & 0.75 & 0.56 & $2.61(3.31)$ & \multirow{3}{*}{0.597} \\
\hline Vigilante & 300 & 0.81 & 0.67 & $2.11(3.33)$ & \\
\hline Inquieto & 300 & 0.65 & 0.42 & $1.40(2.70)$ & \\
\hline
\end{tabular}

Nota: Las cargas del Factor 7 superan el estándar de.40 con comunalidades $>.30$, pero se elimina porque no alcanza el estándar de fiabilidad de.70.

Fuente: Elaboración propia. 
Cuadro 8

EMOCIONES VIGILANTE SOBRE LA VISITA A LA BODEGA

\begin{tabular}{|l|c|c|c|c|c|}
\hline Emociones & $\mathbf{N}$ & $\begin{array}{c}\text { Factor } \\
\mathbf{8}\end{array}$ & Comunalidades & $\mathbf{M}(\mathbf{D E})$ & $\begin{array}{c}\text { Alfa de } \\
\text { Cronbach }\end{array}$ \\
\hline Alerta & 300 & 0.7 & 0.49 & $2.46(3.35)$ & \multirow{2}{*}{0.59} \\
\cline { 1 - 4 } Vigilante & 300 & 0.85 & 0.72 & $2.28(3.45)$ & \\
\cline { 1 - 4 } Inquieto & 300 & 0.66 & 0.43 & $1.45(2.73)$ & \\
\hline
\end{tabular}

Nota: Las cargas del Factor 8 superan el estándar de.40 con comunalidades $>.30$, pero se elimina porque no alcanza el estándar de fiabilidad de.70.

Fuente: Elaboración propia.

Cuadro 9

EMOCIONES VIGILANTE SOBRE LA NOTICIA DEL VINO

\begin{tabular}{|l|c|c|c|c|c|}
\hline Emociones & $\mathbf{N}$ & $\begin{array}{c}\text { Factor } \\
\mathbf{9}\end{array}$ & Comunalidades & $\mathbf{M}(\mathbf{D E})$ & $\begin{array}{c}\text { Alfa de } \\
\text { Cronbach }\end{array}$ \\
\cline { 1 - 5 } Alerta & 300 & 0.69 & 0.48 & $2.65(3.48)$ & \multirow{2}{*}{0.629} \\
\cline { 1 - 4 } Vigilante & 300 & 0.83 & 0.69 & $2.04(3.31)$ & \\
\cline { 1 - 4 } Inquieto & 300 & 0.75 & 0.56 & $1.28(2.58)$ & \\
\hline
\end{tabular}

Nota: Las cargas del Factor 9 superan el estándar de.40 con comunalidades $>.30$, pero se elimina porque no alcanza el estándar de fiabilidad de.70.

Fuente: Elaboración propia.

Al concluir el análisis de componentes principales, se decidió llevar adelante el análisis de intención de compra y diferencias con base al género utilizando las dimensiones que corresponden a emociones positivas y emociones negativas respecto a la oferta de vino, la visita a la bodega y la noticia general sobre el vino. Se decidió omitir el uso de las emociones de vigilancia que corresponden a los factores que fueron eliminados mediante el análisis de componentes y de consistencia interna.

De esta manera, para llevar a cabo el análisis de intención de compra, se tomaron en consideración las respuestas al reactivo \#1 si pudiera, intentaría adquirir el vino de la oferta, así como las respuestas a cada una de las variables que corresponden a emociones positivas respecto a la oferta de vino, visita a la bodega y noticia sobre el vino, para realizar un análisis de regresión. Asimismo, se tomaron en cuenta las respuestas al mismo reactivo mencionado y las respuestas a cada uno de los reactivos que corresponden a emociones negativas a la oferta de vino, visita a la bodega y noticia sobre el vino para realizar el análisis de regresión. Los resultados se muestran de la Figura 3 a la Figura 8. 
Una vez efectuado el análisis de regresión, se determinó realizar la comparación de medias de los participantes de género femenino y masculino con base a los resultados de las dimensiones emociones positivas y emociones negativas. La dimensión sobre emociones de vigilancia fue descartada debido a que no cumplió con los estándares de consistencia interna. El análisis fue llevado a cabo empleando una prueba $t$ de pares intendentes donde la variable independiente fue el género de los participantes y las variables dependientes las emociones positivas y negativas respecto a la oferta de vino, la visita a la bodega y la noticia sobre el vino. Los resultados de la prueba t de pares independientes se ilustra en el Cuadro 10 presentado en la sección de Resultados.

\section{RESULTADOS}

\subsection{Intención de compra y emociones positivas sobre el vino}

Los resultados del análisis de regresión permitieron emitir los siguientes hallazgos: se rechazan las hipótesis nulas Ho: No existe relación entre intención de compra y emociones positivas sobre la oferta de vino (Figura 3), Ho: No existe relación entre intención de compra y emociones positivas sobre la visita a la bodega (Figura 4), y Ho: No existe relación entre la intención de compra y emociones positivas sobre la noticia del vino (Figura 5).

\section{Figura 3 \\ INTENCIÓN DE COMPRA Y EMOCIONES POSITIVAS SOBRE LA OFERTA DE VINO}

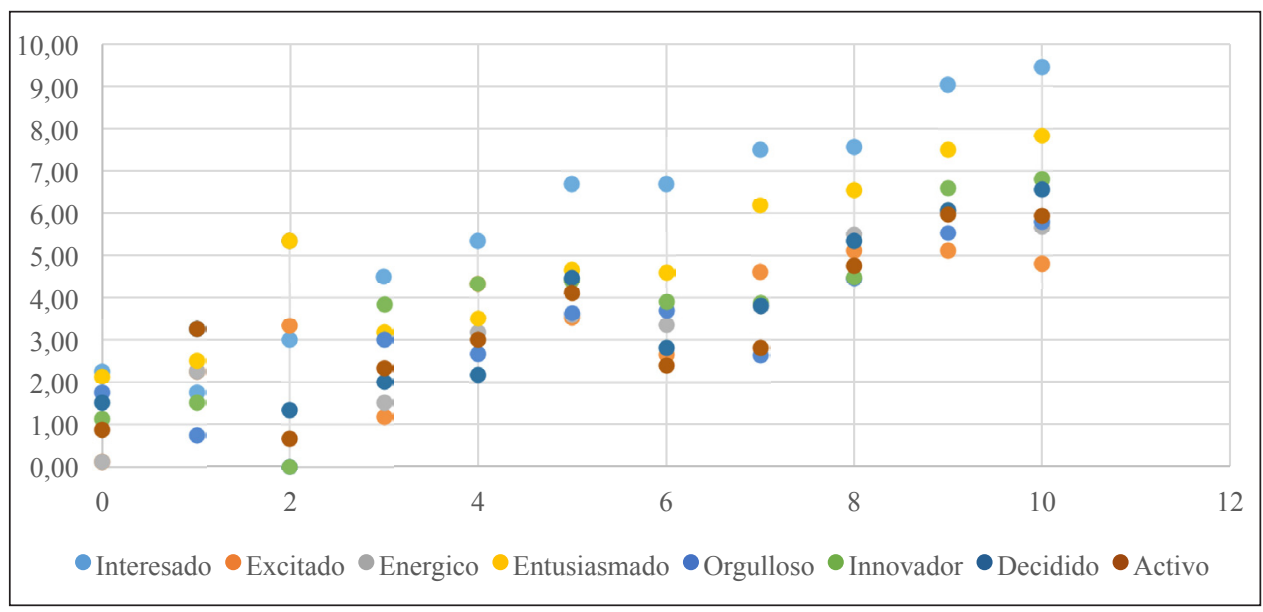

Nota 1: La gráfica de dispersión muestra que existe una relación lineal positiva entre la variable dependiente (intención de compra) y la variable independiente (emociones positivas sobre el vino).

Nota 2: $\mathrm{R}^{2}=.49, \mathrm{p}<.001$.

Fuente: Elaboración propia. 


\section{Figura 4 \\ INTENCIÓN DE COMPRA Y EMOCIONES POSITIVAS SOBRE LA VISITA A \\ LA BODEGA}

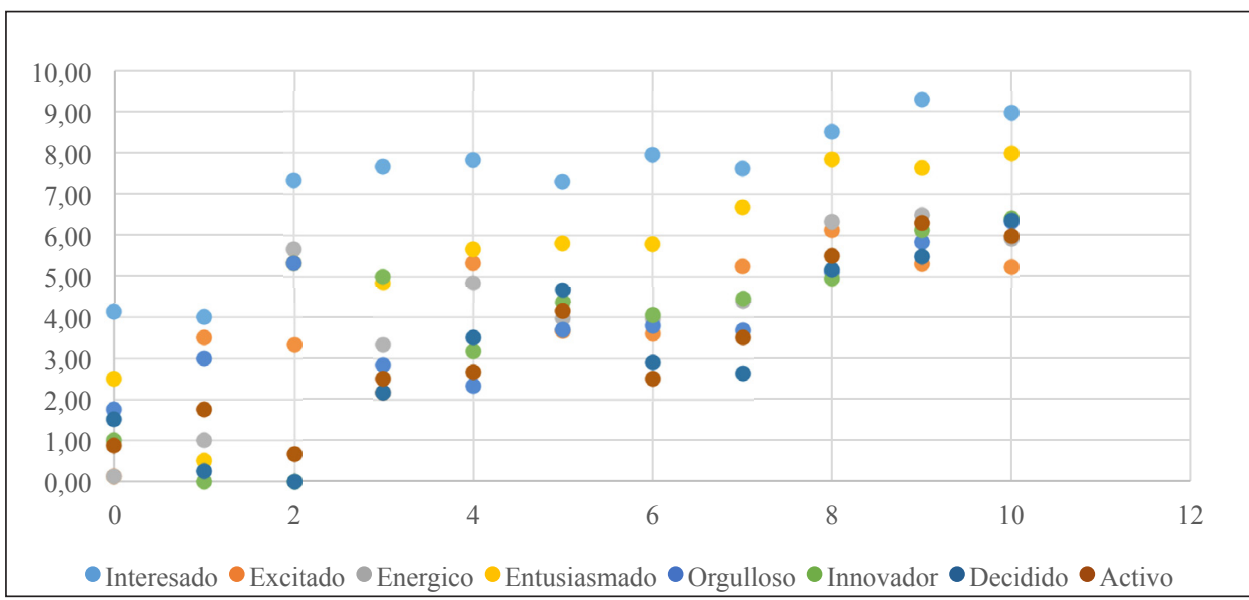

Nota 1: La gráfica de dispersión muestra que existe una relación lineal positiva entre la variable dependiente (intención de compra) y la variable independiente (emociones positivas sobre visita a la bodega).

Nota $2: \mathrm{R}^{2}=.25, \mathrm{p}<.001$.

Fuente: Elaboración propia.

Figura 5

INTENCIÓN DE COMPRA Y EMOCIONES POSITIVAS SOBRE LA NOTICIA DEL VINO

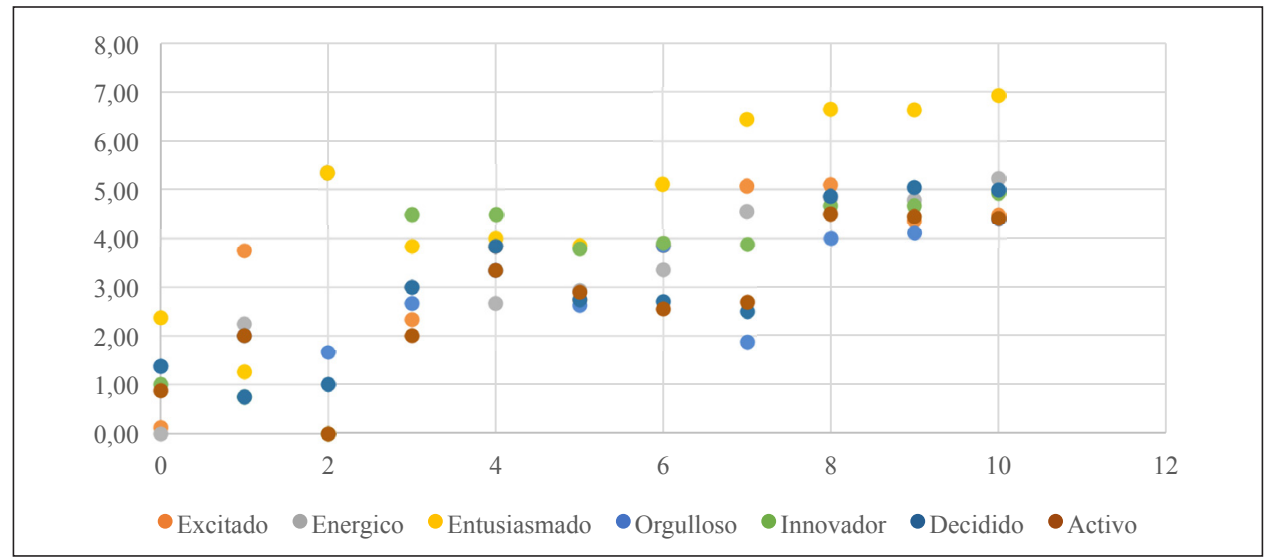

Nota 1: La gráfica de dispersión muestra que existe una relación lineal positiva entre la variable dependiente (intención de compra) y la variable independiente (emociones positivas sobre visita a la bodega).

Nota 2: $\mathrm{R}^{2}=.15, \mathrm{p}<.001$.

Fuente: Elaboración propia. 


\subsection{Intención de compra y emociones negativas sobre el vino}

Respecto a las emociones negativas en relación al vino, se aceptan las hipótesis nulas Ho: No existe relación entre intención de compra y emociones negativas sobre la oferta de vino (Figura 6), Ho: No existe relación entre intención de compra y emociones negativas sobre la visita a la bodega (Figura 7), y Ho: No existe relación entre intención de compra y emociones negativas sobre la noticia del vino (Figura 8).

\section{Figura 6 \\ INTENCIÓN DE COMPRA Y EMOCIONES NEGATIVAS SOBRE LA OFERTA DE VINO}

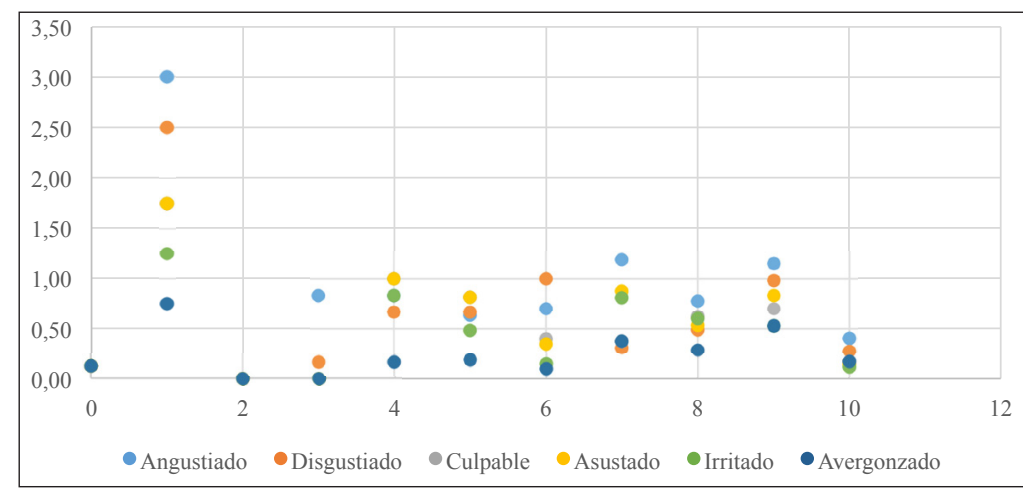

Nota 1: La gráfica de dispersión muestra que no existe una relación lineal entre la variable dependiente (intención de compra) y la variable independiente (emociones negativas sobre el vino). Nota $2: \mathrm{R}^{2}=.02, \mathrm{p}>.10$. Fuente: Elaboración propia.

\section{Figura 7}

\section{INTENCIÓN DE COMPRA Y EMOCIONES NEGATIVAS SOBRE LA VISITA A LA BODEGA}

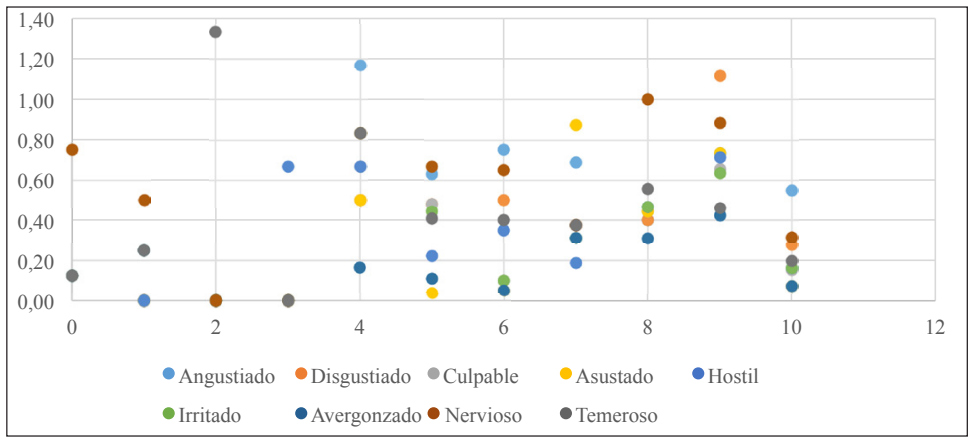

Nota 1: La gráfica de dispersión muestra que no existe una relación lineal entre la variable dependiente (intención de compra) y la variable independiente (emociones negativas sobre la visita a la bodega). Nota 2: $\mathrm{R}^{2}=.01, \mathrm{p}>.10$. Fuente: Elaboración propia. 


\section{Figura 8 \\ INTENCIÓN DE COMPRA Y EMOCIONES NEGATIVAS SOBRE LA NOTICIA DEL VINO}

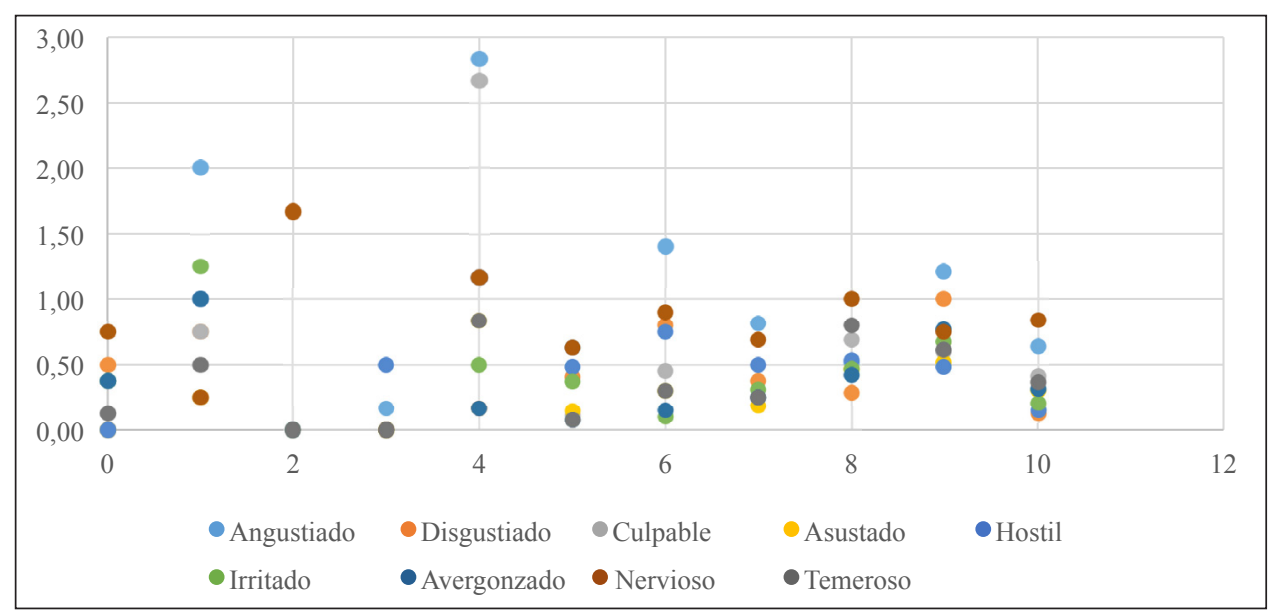

Nota 1: La gráfica de dispersión muestra que no existe una relación lineal entre la variable dependiente (intención de compra) y la variable independiente (emociones negativas sobre la noticia del vino).

Nota 2: $\mathrm{R}^{2}=.03, \mathrm{p}>.10$.

Fuente: Elaboración propia.

\subsection{Emociones positivas y negativas respecto al género}

Para completar el análisis, se hizo la comparación de emociones positivas y emociones negativas entre personas del género femenino y masculino a través de prueba $t$ de pares independientes, lo cual arrojó que los resultados expuestos en el Cuadro 10 sugieren que existen diferencias significativas entre participantes de ambos géneros respecto a emociones negativas sobre la oferta de vino y la visita a la bodega. Las conclusiones completas de las pruebas de hipótesis se presentan en el Cuadro 11.

\section{Cuadro 10}

EMOCIONES POSITIVAS Y NEGATIVAS Y SU RELACIÓN CON EL GÉNERO

\begin{tabular}{|l|c|c|c|c|c|}
\hline Dimensiones & Género & $\mathbf{N}$ & $\mathbf{M}(\mathbf{D E})$ & $\mathbf{t}$ (dos colas) & $\mathbf{p}$ (dos colas) \\
\hline \multirow{2}{*}{$\begin{array}{l}\text { Emociones positivas sobre } \\
\text { vino }\end{array}$} & Femenino & 154 & $39.73(19.63)$ & .29 & $>.10$ \\
\cline { 2 - 4 } & Masculino & 146 & $39.08(18.99)$ & .29 & $>10$ \\
\cline { 1 - 4 } $\begin{array}{l}\text { Emociones positivas sobre } \\
\text { visita }\end{array}$ & Femenino & 154 & $44.49(22.50)$ & .24 & $>.10$ \\
\cline { 1 - 4 } & Masculino & 146 & $43.88(21.96)$ & & \\
\hline
\end{tabular}




\begin{tabular}{|c|c|c|c|c|c|}
\hline Dimensiones & Género & $\mathbf{N}$ & $\mathbf{M}(\mathbf{D E})$ & $\mathrm{t}(\mathrm{dos}$ colas $)$ & $p($ dos colas $)$ \\
\hline \multirow{2}{*}{$\begin{array}{l}\text { Emociones positivas sobre } \\
\text { noticia }\end{array}$} & Femenino & 152 & $26.48(18.18)$ & \multirow{2}{*}{.06} & \multirow{2}{*}{$>.10$} \\
\hline & Masculino & 146 & $26.35(17.55)$ & & \\
\hline \multirow{2}{*}{$\begin{array}{l}\text { Emociones negativas } \\
\text { sobre vino }\end{array}$} & Femenino & 154 & $2.01(5.21)$ & \multirow{2}{*}{-2.07} & \multirow{2}{*}{$<.05$} \\
\hline & Masculino & 146 & $3.63(8.12)$ & & \\
\hline \multirow{2}{*}{$\begin{array}{l}\text { Emociones negativas } \\
\text { sobre visita }\end{array}$} & Femenino & 154 & $6.64(6.52)$ & \multirow{2}{*}{-1.89} & \multirow{2}{*}{$>.05$} \\
\hline & Masculino & 146 & $4.46(9.93)$ & & \\
\hline \multirow{2}{*}{$\begin{array}{l}\text { Emociones negativas } \\
\text { sobre noticia }\end{array}$} & Femenino & 154 & $3.92(8.34)$ & \multirow{2}{*}{-1.10} & \multirow{2}{*}{$>.10$} \\
\hline & Masculino & 146 & $5.14(10.73)$ & & \\
\hline
\end{tabular}

Nota 1: Se compararon los promedios por género (variable independiente) y emociones positivas y negativas con sus factores correspondientes (variables dependientes).

Nota 2: Solamente emociones negativas respecto a la oferta de vino mostraron diferencias significativas por género.

Fuente: Elaboración propia.

\section{Cuadro 11 \\ PRUEBAS DE HIPÓTESIS}

\begin{tabular}{|l|l|}
\hline Hipótesis Nula & Resultado \\
\hline $\begin{array}{l}\text { Ho: No hay relación entre intención de compra y emociones positivas sobre } \\
\text { la oferta de vino. }\end{array}$ & Rechazada \\
\hline $\begin{array}{l}\text { Ho: No existe relación entre intención de compra y emociones positivas } \\
\text { sobre la visita a la bodega. }\end{array}$ & Rechazada \\
\hline $\begin{array}{l}\text { Ho: No existe relación entre la intención de compra y emociones positivas } \\
\text { sobre la noticia del vino. }\end{array}$ & Rechazada \\
\hline $\begin{array}{l}\text { Ho: No existe relación entre intención de compra y emociones negativas } \\
\text { sobre la oferta de vino. }\end{array}$ & Aceptada \\
\hline $\begin{array}{l}\text { Ho: No existe relación entre intención de compra y emociones negativas } \\
\text { sobre la visita a la bodega. }\end{array}$ & Aceptada \\
\hline $\begin{array}{l}\text { Ho: No existe relación entre intención de compra y emociones negativas } \\
\text { sobre la noticia del vino. }\end{array}$ & Aceptada \\
\hline $\begin{array}{l}\text { Ho: No existe relación respecto a emociones positivas sobre la oferta de } \\
\text { vino entre participantes del género femenino y masculino. }\end{array}$ & Aceptada \\
\hline $\begin{array}{l}\text { Ho: No existe relación respecto a emociones positivas sobre la visita a la } \\
\text { bodega entre participantes del género femenino y masculino. }\end{array}$ & Aceptada \\
\hline $\begin{array}{l}\text { Ho: No existe relación respecto a emociones positivas sobre la noticia del } \\
\text { vino entre participantes del género femenino y masculino. }\end{array}$ & Aceptada \\
\hline
\end{tabular}




\begin{tabular}{|l|c|}
\hline Hipótesis Nula & Resultado \\
\hline $\begin{array}{l}\text { Ho: No existe relación respecto a emociones negativas sobre la oferta de } \\
\text { vino entre participantes del género femenino y masculino. }\end{array}$ & Rechazada \\
\hline $\begin{array}{l}\text { Ho: No existe relación respecto a emociones negativas sobre la visita a la } \\
\text { bodega entre participantes del género femenino y masculino. }\end{array}$ & Aceptada \\
\hline $\begin{array}{l}\text { Ho: No existe relación respecto a emociones negativas sobre la noticia del } \\
\text { vino entre participantes del género femenino y masculino. }\end{array}$ & Aceptada \\
\hline
\end{tabular}

Fuente: Elaboración propia.

\section{CONCLUSIONES E IMPLICACIONES}

La presente investigación compara la influencia de las emociones producidas por la oferta de vino, la visita a la bodega y una noticia general sobre el vino en la compra de vino en la bodega entre enoturistas que acuden a un destino del nuevo mundo del vino desde una visión de género, como es Baja California, México, esto presentado como resultado preliminar de un trabajo más extenso entre las regiones del vino que se encuentra replicándose. La elección de esta primera región para la realización del estudio empírico se fundamenta en la representatividad que esta zona tiene dentro del entorno vitivinícola. A este respecto, se observa que existen diferencias entre género en uno de los factores, lo que es coherente con el resultado de Kolyesnikova et al. (2009), en la que encuentra una mayor orientación hacia los sentimientos en los enoturistas de un género que de otro y reafirma los trabajos que consideran la respuesta emocional a la compra es distinta entre géneros (Collado et al., 2013; Dutta et al., 2007; Galvez et al., 2015; Gill et al., 2007).

Los resultados anteriores también matizan las investigaciones de Bastos et al. (2017) y Langner et al. (2014) en los que encuentran similitudes en los consumidores de diferente género, en el sentido que se comenta a continuación. Existe influencia de las emociones en la intención de compra de los consumidores, específicamente emociones positivas respecto a la oferta de vino, la visita a la bodega y la noticia sobre el vino. Estos resultados tienen importantes implicaciones académicas, ya que al referirse al efecto positivo en la intención de compra, se deja ver su estrecha relación con las investigaciones de Kolyesnikova et al. (2009), Lin (2015), Bastos et al. (2017) y otros como Gutjar et al. (2015) y Organ et al. (2015) sobre el consumo de alimentos y bebidas.

Por otra parte, las emociones negativas no tienen un efecto significativo en ninguno de los tres tipos de emociones (vino, visita y noticia) en el destino del nuevo mundo del vino (Baja California, México). Las emociones negativas no pueden utilizarse para predecir la intención de compra de los enoturistas, es de notarse que se encontraron diferencias significativas entre personas de género femenino y masculino en uno de los factores (emociones negativas sobre el vino). De acuerdo a estos resultados, las personas de género masculino tienden a tener mayor incidencia de emociones negativas respecto a la oferta de vino que las personas del género femenino. Este resultado contradice 
las investigaciones de Han et al. (2007) y Schwarz (2000), en los que se considera la influencia negativa de las emociones negativas en la intención de compra, pero, en contraparte, refuerzan los estudios de Olarte-Pascual et al. (2016) y Pelegrín-Borondo (2017), en los que se establecía una mayor influencia de las emociones positivas que negativas en la intención de compra.

En cuanto a las implicaciones empresariales, para comerciantes de vino se sugiere destinar mayor inversión en estrategias de promoción para la creación de una marca que capitalice sobre las cualidades positivas de los vinos que venden, de tal manera que generen experiencias de compra memorables en las bodegas (in situ); y, también, promover prácticas de relaciones públicas encaminadas a la generación de cobertura mediática positiva sobre productos y marcas. Referente al género, se sugiere que los comerciantes impulsen estrategias de marca para el vino que consideren la adecuada segmentación delimitada por la variable género para la oferta de productos y servicios en la bodega, ya que como se encontró en este estudio las emociones influyen de manera distinta entre el sexo femenino y masculino.

Así, hechas las consideraciones anteriores, se ha permitido observar que tanto para las emociones producidas por el vino, la visita y la noticia, las emociones positivas afectan la intención de compra de los enoturistas. En este sentido, aunque con cierta limitación, se puede afirmar que el género es una variable moderadora que influencia las emociones en la intención de compra de vino en la bodega. También, es preciso destacar que en futuras investigaciones se compare la influencia del precio percibido en las emociones producidas en el enoturistas, así como el efecto de mostrar una noticia tanto a favor como en contra (positiva y negativa) en el consumo de vino, ya que puede ejercer efectos distintos. Igualmente, se prevé realizar esta comparación con otros destinos del Nuevo y Viejo mundo del vino, específicamente en California (Estados Unidos) y Rioja (España) al ser considerados destinos enoturísticos importantes y de gran prestigio en cuanto a elaboración y comercialización de vino se refiere (Romano y Natilli, 2010; Wine Institute, 2016). De tal modo que a través de esta comparación se obtengan a la vez dos derivaciones: (i) para confirmar la influencia de las variables que se seleccionaron aplicando el instrumento en más de una bodega, y (ii) para comparar el comportamiento de las mismas variables pero añadiendo el componente región, visto tanto desde la perspectiva geográfica como desde la perspectiva del arraigo del consumo y producción del vino.

Finalmente, cabe mencionar que la muestra empleada en esta investigación, en la bodega en Baja California (Bodegas de Santo Tomás) y perteneciente al nuevo mundo del vino, el $100 \%$ de los enoturistas han sido norteamericanos, principalmente mexicanos aunque también del Sur de California (Estados Unidos). Por lo que ampliar la muestra en un trabajo cross-cultural seguramente permitirá aportar un mayor conocimiento teórico y práctico al estudio del comportamiento de compra de enoturistas en la bodega. Al mismo tiempo, esta investigación refuerza la actual política turística del Gobierno Federal en beneficio del continuo aumento de la competitividad de los destinos turísticos de México, de manera que se aprovechen los avances tecnológicos y de comunicación existentes a favor de los sectores y subsectores, promoviendo la toma de decisiones e implementación de políticas en turismo. 


\section{REFERENCIAS BIBLIOGRÁFICAS}

ANDERSON, K. (2003): «Wine»s new world», Foreign Policy, pp. 47-54.

ATOUT FRANCE (2010): Tourisme et vin, les clientèles françaises et internationales, les concurrents de la France. Comment rester compétitif? Editions Atout France, Paris.

BAGOZZI, R. (1997): «Goal-directed behaviors in marketing: the role of emotion, volition and motivation», Psychology \& Marketing, vol. 14, n 4, pp. 309-313.

BARRENA, R. y SÁNCHEZ, M. (2012): «Neophobia, personal consumer values and novel food acceptance», Food Quality and Preference, vol. 27, n 1, pp. 72-84.

BASTOS, A.D.F.V., DA COSTA, F.J. y VASCONCELOS, M.M. (2017): «Consumo de bebidas alcoólicas por jovens: implicações para o marketing social», REMark, vol. $16, \mathrm{n}^{\circ} 4$, pp. 469-486.

BAUTZER, D. (2010): Marketing de cidades: construção de identidade, imagen e futuro. Atlas S. A., Sao Paulo.

BENNET, P. y HARREL, G. (1975): «The Role of Confidence in Understanding and Predicting Buyers' Attitudes and Purchase Intentions». Journal of Consumer Research, vol. 2, n 2, pp. 110-117.

BLANCO-GONZÁLEZ, A., MARTÍN-ARMARIO, E. y MERCADO, C. (2013): «La influencia de las actitudes en la intención de compra del coleccionista». Revista Europea de Dirección y Economía de la Empresa, vol. 22, nº 2, pp. 61-68.

BRUWER, J., COODE, M., SALIBA, A. y HERBST, F. (2013): «Wine tourism experience effects of the tasting room on consumer brand loyalty», Tourism Analysis, vol. 18, no 4, pp. 399-414.

BUIL, I., MARTÍNEZ, E. y MONTANER, T. (2012): «La influencia de las acciones de marketing con causa en la actitud hacia la marca», Cuadernos de Economía y Dirección de la Empresa, vol. 15, n 2, pp. 84-93

CELAYA, D. (2014). El desarrollo del sector vitivinícola en Baja California (2000-2013): Un análisis desde la perspectiva del desarrollo endógeno, Tesis Doctoral. El Colegio de la Frontera Norte (México).

CERVANTES, S. (2012): Bodegas de Santo Tomás, una historia añeja del vino. Disponible enhttp://eleconomista.com.mx/estados/2012/07/29/bodegas-santo-tomas-historiaaneja-vino

CHANG, P.L. y CHIENG, M.H. (2006): «Building Consumer-Brand Relationship: A Cross-Cultural Experiential View», Psychology \& Marketing, vol. 23, nº11, pp. 927959.

CHARTERS, S. y ALI-KNIGHT, J. (2002): «Who is the wine tourist?», Tourism Management, vol. 23, no 3, pp. 311-319.

CHEN, G. y HUANG, S.S. (2017): «Toward a theory of backpacker personal development: Cross-cultural validation of the BPD scale», Tourism Management, vol. 59, pp. 630-639.

COLLADO, A.M., RICO, M.G. y TALAYA, Á.E. (2013): «Caracterización de visitantes enológicos en España: un análisis comparativo en cinco denominaciones de origen», Revista de Análisis Turístico, nº 15, pp. 87-97. 
DAS, G. (2014): «Factors affecting Indian shoppers' attitude and purchase intention: An empirical check», Journal of Retailing and Consumer Services, vol. 21, $\mathrm{n}^{\circ}$ 4, pp. 561-569.

DHAR, R. y WERTENBROCH, K. (2000): «Consumer choice between hedonic and utilitarian goods», Journal of Marketing Research, vol. 37, n 1, pp. 60-71.

DUTTA, S., BISWAS, A. y GREWAL, D. (2007): «Low price signal default: an empirical investigation of its consequences», Journal of the Academy Marketing Science, vol. $35, \mathrm{n}^{\circ} 1, \mathrm{pp} .76-88$.

GALVEZ, J.C.P., FERNÁNDEZ, G.A.M. y LÓPEZ-GUZMÁN, T. (2015): «Wine festivals as a vehicle of promotion of a tourist destination», Mediterranean Journal of Social Sciences, vol. 6, no 3 S2, pp. 574- 581.

GETZ, D. y BROWN, G. (2006): «Critical success factors for wine tourism regions: A demand analysis», Tourism Management, vol. 27, $\mathrm{n}^{\circ}$ 1, pp. 146-158.

GILBERT, D. y WONG, R.K.C. (2003): «Passenger expectations and airline service: a Hong Kong based study», Tourism Management, vol. 24, n ${ }^{\circ}$ 5, pp. 519-532.

GILL, D., BYSLMA, B. y OUSCHAN, R. (2007): «Customer perceived value in a cellar door visit: the impact on behavioural intentions», International Journal of Wine, vol. $19, \mathrm{n}^{\mathrm{o}} 4,257-275$.

GRISKEVICIUS, V., SHIOTA, M. y NOWLIS, S.M. (2010): «The many shades of rosecolored glasses: An evolutionary approach to the influence of different positive emotions», Journal of Consumer Research, vol. 37, n 2, pp. 238-250.

GUTJAR, S., DE GRAAF, C., KOOIJMAN, V., DE WIJK, R.A., NYS, A., TER HORST, G.J. y JAGER, G. (2015): «The role of emotions in food choice and liking», Food Research International, vol. 76, pp. 216-223.

HAMARI, J. (2015): «Why do people buy virtual goods? Attitude toward virtual good purchases versus game enjoyment», International Journal of Information Management, vol. 35, nº 3, pp. 299-308.

HAN, S., LERNER, J.S. y KELTNER, D. (2007): «Feeling and consumer decision making: The appraisal-tendency framework», Journal of Consumer Psychology, vol. 17, $\mathrm{n}^{\circ} 3$, pp. 158-168.

HIRSCHMAN, E. y STERN, B.B. (1999): «The roles of emotion in consumer research», Advances in Consumer Research, vol. 26, pp. 4-11.

HORSKA, E., BERCIK, J., KRASNODEBSKI, A., MATYSIK-PEJAS, R. y BAKAYOVA, H. (2016): «Innovative approaches to examining consumer preferences when choosing wines», Agricultural Economics (ZemědělskáEkonomika), vol. 62, pp. 124133.

HOWLEY, M. y VAN WESTERING, J. (2008): «Developing wine tourism: A case study of the attitude of English wine producers to wine tourism», Journal of Vacation Marketing, vol. 14, $\mathrm{n}^{\circ} 1$, pp. 87-95.

ILICIC, J. y WEBSTER, C. (2011): «Effects of multiple endorsements and consumercelebrity attachment on attitude and purchase intention», Australasian Marketing Journal, vol. 19, nº 4, pp. 230-237.

KAEFER, F., HEILLMAN, C.M. y RAMENOFSKY, S.D. (2012): «The impact of a wife's budgetary and romantic attitudes towards luxury goods on their self-purchases 
and purchases made for them by their husbands», Journal of Retailing and Consumer Services, vol. 19, n 3 , pp. 297-303.

KOLYESNIKOVA, N. y DODD, T.H. (2008): «Effects of winery visitor group size on gratitude and obligation», Journal of Travel Research, vol. 47, $\mathrm{n}^{\circ}$ 1, pp. 104-112.

KOLYESNIKOVA, N., DODD, T.H. y WILCOX, J.B. (2009): «Gender as a moderator of reciprocal consumer behavior», Journal of Consumer Marketing, vol. 26, $\mathrm{n}^{\circ} 3$, pp. 200-213.

LABROO, A.A. y PATRICK, V.M. (2009): «Psychological distancing: Why happiness helps you see the big picture», Journal of Consumer Research, vol. 35, n 5, pp. 800-809.

LABROO, A.A. y RUCKER, D.D. (2010): «The orientation-matching hypothesis: An emotion-specificity approach to affect regulation», Journal of Marketing Research, vol. 47, nº 5, pp. 955-966.

LANGNER, T., BRUNS, D., FISCHER, A. y ROSSITER, J.R. (2014): «Falling in love with brands: a dynamic analysis of the trajectories of brand love», Marketing Letters, vol. $27, \mathrm{n}^{\circ} 1$, pp. 15-26.

LAROCHE, M., KIM, C. y ZHOU, L. (1996): «Brand Familiarity and Confidence as Determinants of Purchase Intention: An Empirical Test in a Multiple Brand Context», Journal of Business Research, vol. 37, $\mathrm{n}^{\circ}$ 2, pp. 115-120.

LEE, H.L. y YUN, Z.S. (2015): «Consumers' perceptions of organic food attributes and cognitive and affective attitudes as determinants of their purchase intentions toward organic food», Food Quality and Preference, vol. 39, pp. 259-267.

LI, J., CANZIANI, B. y HSIEH, Y. (2016): «US and Chinese perceptions of simulated US courtesy», Worldwide Hospitality and Tourism Themes, vol. 8, n 1, pp. 29-40.

LIN, H.C. (2015): «Moderating roles on individuals' decisions when making choices for others», Food Quality and Preference, vol. 39, pp. 221-227.

LOEBNITZ, N., MUELLER, S. y GRUNERT, K. (2015): «Impacts of situational factor son process attribute uses for food purchases», Food Quality and Preference, vol. 44, pp. 84-91.

LU, L.C., CHANG, W.P. y CHANG, H.H. (2014): «Consumer attitudes toward bloggers's sponsored recommendations and purchase intention: The effect of sponsorship type, product type, and brand awareness», Computer in Human Behavior, vol. 34, pp. 258-266.

MATUTE, J., POLO, Y. y UTRILLAS, A. (2015): «Las características del boca-oído electrónico y su influencia en la intención de recompra online», Revista Europea de Dirección y Economía de la Empresa, vol. 24, nº 2, pp. 61-75.

MIAO, L., MATTILA, A. y MOUNT, D. (2011): «Other consumer in service encounters: A script theoretical perspective», International Journal of Hospitality Management, vol. 30, nº 4, pp. 933-941.

NIE, P., ZHAO, X., YU, L., WANG, C. y ZHANG, Y. (2015): «Social Emotion Analysis System for Online News», en Web Information System and Application Conference (WISA), 2015 12th (pp. 43-48). IEEE.

OLARTE-PASCUAL, C., PELEGRÍN-BORONDO, J. y REINARES-LARA, E. (2016): «Cognitive-affective model of acceptance of mobile phone advertising», E+M Ekonomie a Management, vol. 19, nº, pp. 134-148. 
ORGAN, K., KOENIG-LEWIS, N., PALMER, A. y PROBERT, J. (2015): «Festivals as agents for behavior change: A study of food festivals engagement and subsequent food choices», Tourism Management, vol. 48, pp. 84-99.

ORTA, M., OLAGUE, J.T., LOBO, M.O. y CRUZ, I. (2016): «Importance and assessment of satisfaction components in the oenological experience in the Guadalupe Valley, Ensenada, Baja California: Contributions to the sustainability management process», Revista de Análisis Turístico, vol. 22, n 2, pp. 39-55.

PELEGRÍN-BORONDO, J. (2017): Instrumento de medición, Universidad de La Rioja (España).

PELEGRÍN-BORONDO, J., REINARES-LARA, E. y OLARTE-PASCUAL, C. (2017): «Assessing the acceptance of technological implants (the cyborg): Evidences and challenges», Computers in Human Behavior, vol. 70, pp. 104-112.

PELEGRÍN-BORONDO, J., ARIAS-OLIVA, M. y OLARTE-PASCUAL, C. (2016): «Emotions, price and quality expectations in hotel services», Journal of Vacation Marketing, vol. 21, n 4, pp. 351-355.

PELEGRÍN-BORONDO, J., JUANEDA-AYENSA, E., GONZÁLEZ-MENORCA, L. y GONZÁLEZ-MENORCA, C. (2015): «Dimensions and basic emotions. A complementary approach to the emotions produced to tourists by the hotel», Journal of Vacation Marketing, vol. 21, n ${ }^{\circ}$ 4, pp. 351-365.

PENZ, E. y HOGG, M.K. (2011): «The role of mixed emotions in consumer behaviour», European Journal of Marketing, vol. 45, n 1-2, pp. 104-132.

PRATT, M. (2014): «Four Wine Tourist Profiles», en Academy of Wine Business Research, $8^{\text {th }}$ International Conference, Geisenheim (Alemania).

QUINTAL, V.A., THOMAS, B. y PHAU, I. (2015): «Incorporating the winescape into the theory of planned behaviour: Examining 'new world' wineries», Tourism Management, vol. 46, pp. 596-609.

ROMANO, M.F. y NATILLI, M. (2010): «Wine tourism in Italy: New profiles, styles of consumption, ways of touring», Turizam: znanstveno-stručničasopis, vol. 57, nº 4, pp. 463-475.

RUIZ, A.V., RIAÑO, C. y DI PERRI, C. (2014): «Do low price signals influence online purchases of tourist accommodation services? The moderating role of gender», Business and Management Research, vol. 3, nº 4, pp. 96- 111.

RUSSELL, J.A. (2003): «Core affect and psychological construction of emotion», Psychological Review, vol. 110, $\mathrm{n}^{\mathrm{o}} 1$, pp. 145-172.

SCHERER, K.R. (2005): «What are emotions? And how can they be measured?», Social Science Information, vol. 44, no 4, pp. 695-729.

SCHWARZ, N. (2000): «Emotion, cognition, and decision making», Cognition and Emotion, vol. 14, no 4, pp. 433-440.

SIEGRIST, M. (2008): «Factors influencing public acceptance of innovative food technologies and products», Trends in Food Science \& Technology, vol. 19, n 11, pp. 603-608.

SILVA, A.P., JAGER, G., VAN BOMMEL, R., VAN ZYL, H., VOSS, H.P., HOGG, T., PINTADO, M. y DE GRAAF, C. (2016): «Functional or emotional? How Dutch and Portuguese conceptualize beer, wine and non-alcoholic beer consumption», Food Quality and Preference, vol. 49, pp. 54-65. 
SPARKS, B. (2007): «Planning a wine tourism vacation? Factors that help to predict tourist behavioural intentions», Tourism Management, vol. 28, nº 5, pp. 1180-1192.

STEVENS, B.F. (1992): «Price value perceptions of travelers», Journal of Travel Research, vol. 31, no 2 , pp. 44-48.

SUH, Y.K. y McAVOY, L. (2005): «Preferences and trip expenditures - a conjoint analysis of visitors to Seoul, Korea», Tourism Management, vol. 26, nº 3, pp. 325-333.

SULTAN, F. y SIMPSON, M.C. (2000): «International service variants: Airline passenger expectations and perceptions of service quality», Journal of Services Marketing, vol. $14, n^{\circ} 3$, pp. 188-216.

TENG, L. (2009): «A comparison of two types of price discounts in shifting consumers' attitudes and purchase intentions», Journal of Business Research, vol. 62, no 1, pp. 14-21.

TREJO-PECH, C.O., LÓPEZ-REYNA, C., HOUSE, L.A. y SARMIENTO-PÉREZ, F.J. (2010): «Globalization in the Wine Industry and the Case of Baja California Mexico», en $20^{\text {th }}$ Annual World Food and Agribusiness Forum and Symposium 2010, Boston (USA).

VANHOVE, N. (2005): The Economics of Tourism Destinations. Elsevier, Oxford.

VILLANUEVA, E. (2014): «La innovación como causa del éxito exportador vinícola del Nuevo Mundo Anglosajón», Revista Iberoamericana de Viticultura, Agroindustria y Ruralidad, vol. 1, $\mathrm{n}^{\mathrm{O}}$ 1, pp. 1-12.

WANG, X., YU, C. y WEI, Y. (2012): «Social Media Peer Communication and Impacts on Purchase Intentions: A Consumer Socialization Framework», Journal of Interactive Marketing, vol. 26, $\mathrm{n}^{\mathrm{O}}$ 4, pp. 198-208.

WEINER, B. (1985): «An attributional theory of achievement, motivation, and emotion», Psychological Review, vol. 92, $\mathrm{n}^{\circ}$ 4, pp. 548-573.

WINE INSTITUTE (2016). The Economic Impact of the California Wine and Winegrape Industry, 2015.Disponible en http://www.wineinstitute.org/files/Wine_Institute_2015_ Economic_Impact_Highlights.pdf

YEH, S.S., CHEN, C. y LIU, Y.C. (2012): «Nostalgic emotion, experiential value, destination image, and place attachment of cultural tourists», Advances in hospitality and leisure, vol. 8, pp. 167-187.

YEH, T.M. y JENG, M.Y. (2015): «The visiting motivation, perceived value and future behavioural intentions of winery tourists», International Journal of Services and Operations Management, vol. 21, $\mathrm{n}^{\mathrm{o}}$ 3, pp. 354-369.

YU, L., ZHIFAN, Y., NIE, P., ZHAO, X. y ZHANG, Y. (2015): «Multi-source Emotion Tagging for Online News», en Web Information System and Application Conference (WISA), 2015 12th (pp. 49-52). IEEE.

YUAN, J., MORRISON, A.M., CAI, L.A. y LINTON, S. (2008): «A model of wine tourist behaviour: a festival approach», International Journal of Tourism Research, vol. 10, no 3, pp. 207-219.

ZAMORA, J., VÁSQUEZ, A, MORALES, F., GUIBERT, N. y ARCE, O. (2006): «¿Existe lealtad en las compras de vino? Un estudio exploratorio de la racionalidad del comprador», en VII Seminario Iberoamericano: Viticultura y Ciencias Sociales, Universidad de Talca. Talca (Chile). 